\title{
Electronic Transport Properties of Phenalenyl Molecular Devices via Gated Modulation
}

\author{
Xiaohui Jiang*, Wen Liu, Jianhua Zhao \\ Department of Physics and Information Engineering, Jining University, Qufu, China \\ Email: *xiaohuijiang94@163.com
}

How to cite this paper: Jiang, X.H., Liu, W. and Zhao, J.H. (2021) Electronic Transport Properties of Phenalenyl Molecular Devices via Gated Modulation. Journal of Applied Mathematics and Physics, 9, 503514.

https://doi.org/10.4236/jamp.2021.93035

Received: October 19, 2020

Accepted: March 28, 2021

Published: March 31, 2021

Copyright $\odot 2021$ by author(s) and Scientific Research Publishing Inc. This work is licensed under the Creative Commons Attribution International License (CC BY 4.0).

http://creativecommons.org/licenses/by/4.0/

\section{Open Access}

\begin{abstract}
By applying nonequilibrium Green's functions (NEGF) in combination with the density functional theory (DFT), we investigate the electronic transport properties of gated phenalenyl molecular devices with two different contact geometries. The calculated results show that electronic transport properties of the two different devices can be modulated by external transverse gates. When the molecule contacts the Au electrodes through two second-nearest sites, the current-voltage $(I-V)$ characteristic curves are symmetric and suppressed by the gate electrodes. However, a rectifying behavior will occur when the electrodes connect the molecule on both sides, one second-nearest site and one third-nearest site, respectively. Mechanisms for such phenomena are proposed and these findings suggest a new opportunity for developing molecular devices.
\end{abstract}

\section{Keywords}

Electronic Transport, Transverse Gate, Nonequilibrium Green's Function, Phenalenyl Molecular

\section{Introduction}

Since the use of individual molecules as functional electronic devices was suggested in 1974 [1], the advances of nanotechnology have led to the fabrication of various molecular devices based on mono-layer arrays of molecules [2] [3] [4] [5]. In the last several years, many experimental and theoretical works were carried out to study the transport properties of the single molecules, to make further efforts to design the molecular electronic devices. Recently, there are two main ways to control the charge transport properties effectively. One is through the conformational changes in the molecular junctions themselves, such as depending on the lattice orientation, the different anchor position to the elec- 
trodes, the contact distance between the molecule and electrode, the different contact geometries of the molecules [6] [7] [8] [9] and so on, and the other way is through the field-effect gating. Many workers have focused their attention on the former for a long time, and achieved many advances [10] [11] [12] [13] [14]. However, in consideration of a low operation frequency in this way, more and more attention is transferred to the latter one due to its high operation frequency. Recently, several experiments have already identified its feasibility [15] [16] [17] [18]. Song et al. investigated the electronic transport properties of $\mathrm{Au}-\mathrm{BDT}-\mathrm{Au}$ and Au-ODT-Au junctions respectively, and the results indicated that the $I$ - $V$ characteristics are significantly dependent on the external gate voltages [16]. $\mathrm{Xu}$ and his coworkers found perylene tetracarboxylic diimide (PTCDI), a redox molecule, could be reversibly controlled with a gate electrode over nearly 3 orders of magnitude at room temperature [18]. On the theoretical aspects, many FET-like models were designed, such as carbon nanotubes-FET [19], nanowires-FET [20], graphene-FET [21], single molecular transistors [22] [23] [24] [25] and so on. Recently, the current behavior of the single molecular has received increasing attention, and many works have shed light on the organic molecular transistors controlled by the transverse field. M. Di Ventra et al. used the first-principle method to calculate the transport properties of a benzene-1,4-dithiolate molecule with a third gate, and the results showed that the resistance of the molecule rises from its zero-gate-bias value to a value roughly equal to the quantum of resistance [23]. Avik W and his coworkers found if we could engineer a large molecular dipole along a suitable direction, the conformational transitions could aid electrostatic gate control significantly [26].

Phenalenyl, a stable organic radical with high symmetry, and its derivatives have attracted much attention for the intriguing properties such as the electrical, optical, and magnetic properties [27] [28] [29]. Fan and his co-workers have investigated the phenalenyl molecular device with different contact geometries, and the $I-V$ curves showed the negative differential resistance and rectifying behaviors [30]. Tagami et al. investigated the quantum transport properties of single phenalenyl-based molecules, and found that changing the sites connected to the electrodes or substituting the central atom of the phenalenyl can tune the transport characteristics [31]. But the electronic properties of the phenalenyl under the transverse gates are still uncertain. In this work, we successfully demonstrate the controllability of the material junction's conductivity by the transverse gates, and the tunneling behaviors induced by the gate, source and drain voltages are investigated systemically.

\section{Models and Methods}

The geometries of the device proposed in this work are illustrated in Figure 1. This system can be divided into three parts: the left electrode, the right electrode and the scattering region. The phenalenyl molecule is connected to two $\mathrm{Au}$ (111) $-(4 \times 4)$ source and drain electrodes with a transverse gate electrode in a FET 


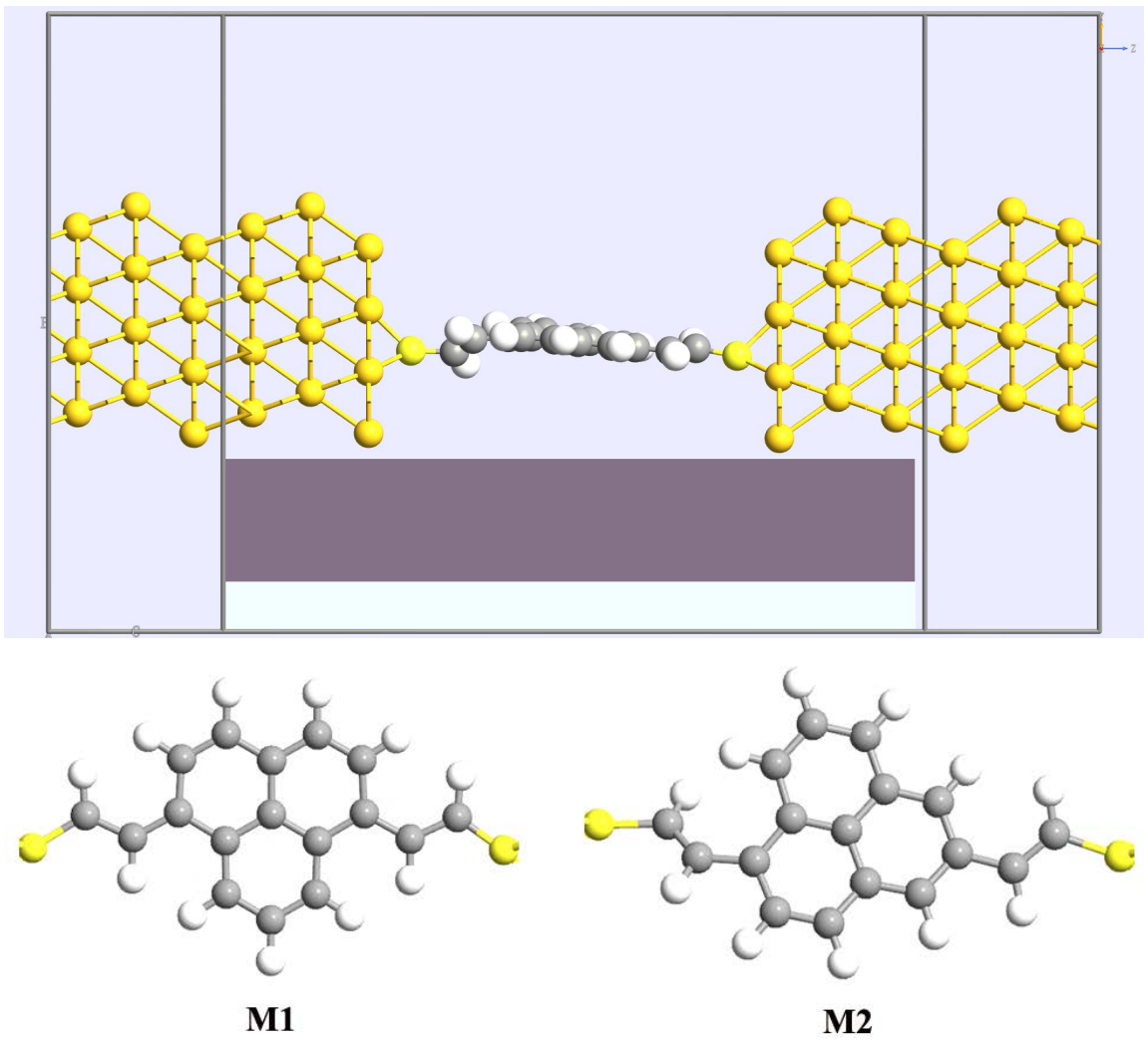

Figure 1. Schematic description of the two single phenalenyl molecular devices: M1 corresponds to the molecule connecting the electrodes via two second-nearest sites from the center atom. M2 corresponds to the molecule connecting the electrodes via one second-nearest site and one third-nearest site from the center atom.

configuration. The sulfur atom is chosen to be located at the hollow site of the gold triangle and the Au-S distance is $2.0 \AA$, which is a typical Au-S distance. M1 and M2 correspond to the two different contacted geometries. In our calculations, the exchange-correlation potential is described by the Perdew-Burke-Ernzerhof parameter of the generalized gradient approximation (GGA.PBE). A single- $\zeta$ (SZ) atomic orbital basis set is employed for $\mathrm{Au}$ atoms and double- $\zeta$ plus polarization (DZP) basis set is adopted for the rest. Before calculating the electron transport properties of the device, all atoms in the channel region are relaxed with a force tolerance of $0.05 \mathrm{eV} / \AA$. The geometrical optimizations and all the calculations are performed using the Atomistix ToolKit (ATK), which is based on fully self-consistent non-equilibrium Green's function (NEGF) and density functional theory (DFT). According to NEGF formulas, the source-drain current $I_{s d}$ through the system is obtained by Landauer-Büttiker formula,

$$
I_{\text {sd }}=\frac{2 e}{h} \int T\left(E, V_{s d}\right)\left[f\left(E-\mu_{L}\right)-f\left(E-\mu_{R}\right)\right] \mathrm{d} E
$$

where $T\left(E, V_{s d}\right)$ is the transmission coefficient of the system at energy $E$ under the source-drain bias $\left(V_{s d}\right), \mu_{L(R)}$ and $f\left(E-\mu_{L(R)}\right)$ are the chemical potential and Fermi function of the left (right) electrode, respectively. For simplicity, the average Fermi level $E_{f}$ of the system is set to zero, thus the current is in fact the 
integral area of transmission curve inside the bias window [-eV/2,eV/2]. The transmission spectrum covers the integrated transmission coefficients $T(E, V, k)$ over the 2D Brillouin zone of incident wave vectors $\boldsymbol{k}$,

$T(E, V)=\frac{1}{\Omega} \int_{\tilde{\Omega}} \mathrm{d} k T(E, V, k)$, where $\Omega$ is the area of the reference unit cell surface.

$$
f(E-\mu)=1 /\left\{1+\exp \left[(E-\mu) /\left(k_{B} T_{\text {temp }}\right)\right]\right\}
$$

The Fermi-Dirac distribution function, $k_{B}$ is Boltzmann constant, and $T_{\text {temp }}$ is the temperature. Furthermore, an additional gate voltage $\left(V_{g}\right)$ is applied on the molecule to extend the device's function. From Figure 1, we can see the gate electrode is not a real physical electrode, and there is no current flowing from the source or drain electrode to the gate electrode. The effect of gate voltage is taken into account by shifting the scattering region part of the Hamiltonian (converted into an electrostatic potential energy). This is equivalent to assume that the gate electrode induces an external potential localized in the scattering region.

\section{Results and Discussion}

The self-consistently calculated $I-V$ characteristics for the molecular device in the $\mathrm{M} 1$ configuration under the bias range from $0 \mathrm{~V}$ to $1.5 \mathrm{~V}$ with the gate voltage $\left(V_{g}\right)$ setting to different values $0.0 \mathrm{~V}, 2.0 \mathrm{~V}, 4.0 \mathrm{~V}, 8.0 \mathrm{~V}$ and $12.0 \mathrm{~V}$ are shown in Figure 2. For the $I$ - $V$ curves are symmetric, we just show the positive bias range. From this figure, we can see controllable gate-voltage dependence in this molecular junction. In the positive $V_{s d}$ region, the gated currents increase gradually slower than that of $V_{g}=0.0 \mathrm{~V}$, which seems the $I$ - $V$ curves are suppressed by the gate electrodes. We present the transmission coefficients at $E_{f}$ are $0.7742,0.4406,0.3759,0.4439$ and 0.0884 for $V_{g}=0.0 \mathrm{~V}, 2.0 \mathrm{~V}, 4.0 \mathrm{~V}, 8.0 \mathrm{~V}$ and $12.0 \mathrm{~V}$ via $V_{s d}=0.0 \mathrm{~V}$, respectively, and it could be found that the transmission coefficients applied gate voltages obviously reduced, which are against the device transport, leading to the suppressed $I$ - $V$ curves in Figure 2.

The particular variation tendency of $I$ - $V$ curve after applying gate voltages can be interpreted in terms of transmission spectrum $T(E)$ and spatial distribution of frontier molecular orbitals. As shown in Figure 3, we can see that as applied positive $V_{g}$ increases, the transmission peaks flow to the low energy, and gradually move out of the bias window. That means the currents are decreasing, as we all know that the current is determined by $T(E, V)$ in the bias window. At $V_{g}=$ $0.0 \mathrm{~V}$, within the bias window, there is a broaden transmission peak around the Fermi level derived from the LUMO $(0.162 \mathrm{eV})$ level, which is the main transmission channel in this device. When $V_{g}$ increases to $2.0 \mathrm{~V}$, the broaden transmission peak is divided into some small and low peaks, and all the peaks are moving to the low energy, in this case, the HOMO resonance is closer to $E_{f}$ as the main transmission channel. To further add the gate voltage to $8.0 \mathrm{~V}$, the HOMO resonance is moving out of the bias window, and while $V_{g}=12.0 \mathrm{~V}$, the 


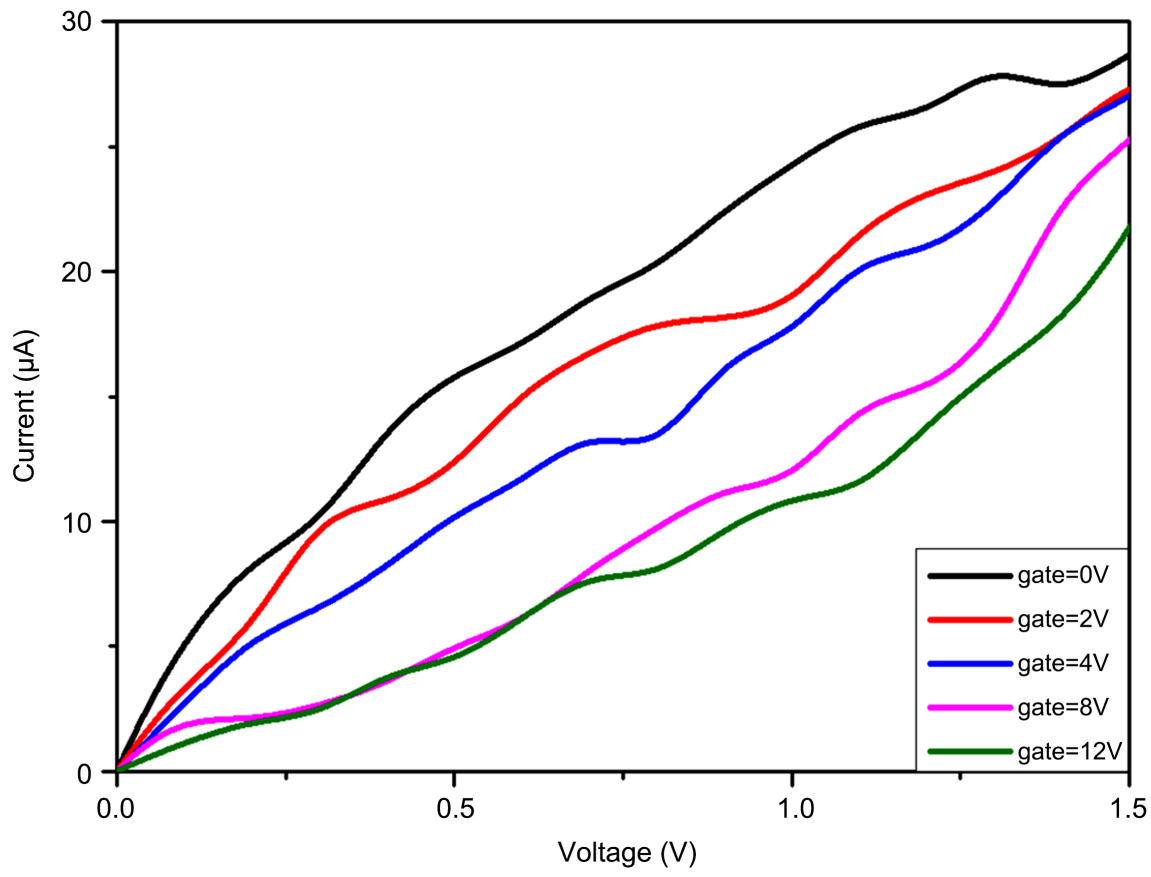

Figure 2. (Color online) Source-Drain currents as a function of $V_{s d}$ in the M1 device configuration for $V_{g}=0.0 \mathrm{~V}, 2.0 \mathrm{~V}, 4.0 \mathrm{~V}, 8.0 \mathrm{~V}$ and $12.0 \mathrm{~V}$, respectively.

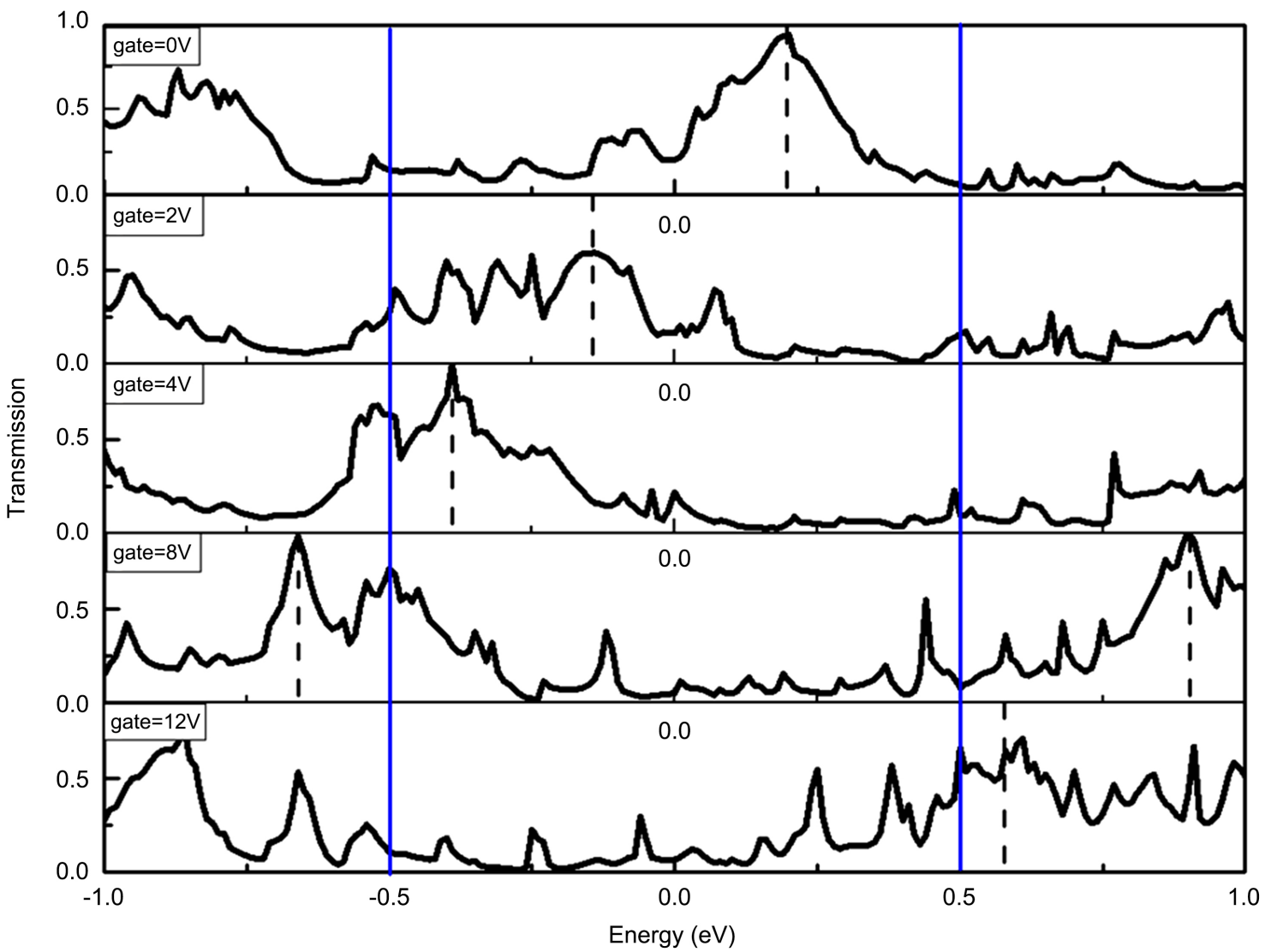

Figure 3. Transmission spectra T(E) at $V_{s d}=1.0 \mathrm{~V}$ for $V_{g}=0.0 \mathrm{~V}, 2.0 \mathrm{~V}, 4.0 \mathrm{~V}, 8.0 \mathrm{~V}$ and $12.0 \mathrm{~V}$ in the M1 device configuration, respectively. The blue solid lines indicate the bias window, and the black dashed lines represent the molecular orbitals. 
broaden transmission peaks are completely out of the bias window, except for some smaller and lower peaks. For this reason, currents are suppressed when we add the positive gate voltages. But from this figure we also can see another molecular orbitals are moving closer to $E_{f}$ as the gates adding, which means if we further add the gate voltages, the current will not depressed enormously for the other resonance will move into the bias window as the main transmission channel.

The self-consistent calculated $I$ - $V$ characteristic for M2 device under the bias range from $-2.0 \mathrm{~V}$ to $2.0 \mathrm{~V}$ with the gate voltage $\left(V_{g}\right)$ setting to different values $0.0 \mathrm{~V}, 2.0 \mathrm{~V}, 4.0 \mathrm{~V}$, and $8.0 \mathrm{~V}$ are shown in Figure 4. We can see that the currents on the positive bias voltages are bigger than that on the negative bias under different gates. It means that the device shows an obviously rectifying performance. In a lower bias range, the currents are almost linear, representing an approximate Ohmic behavior and corresponding to a nonresonant tunneling. As the bias increases, the current changes non-linearly, which suggests the onset of the resonant conduction. What is more, the source-drain currents can be controlled by the gate voltage in the positive bias, and those present in the rise with the applied gate increasing. However, gate voltage has no effect in the negative

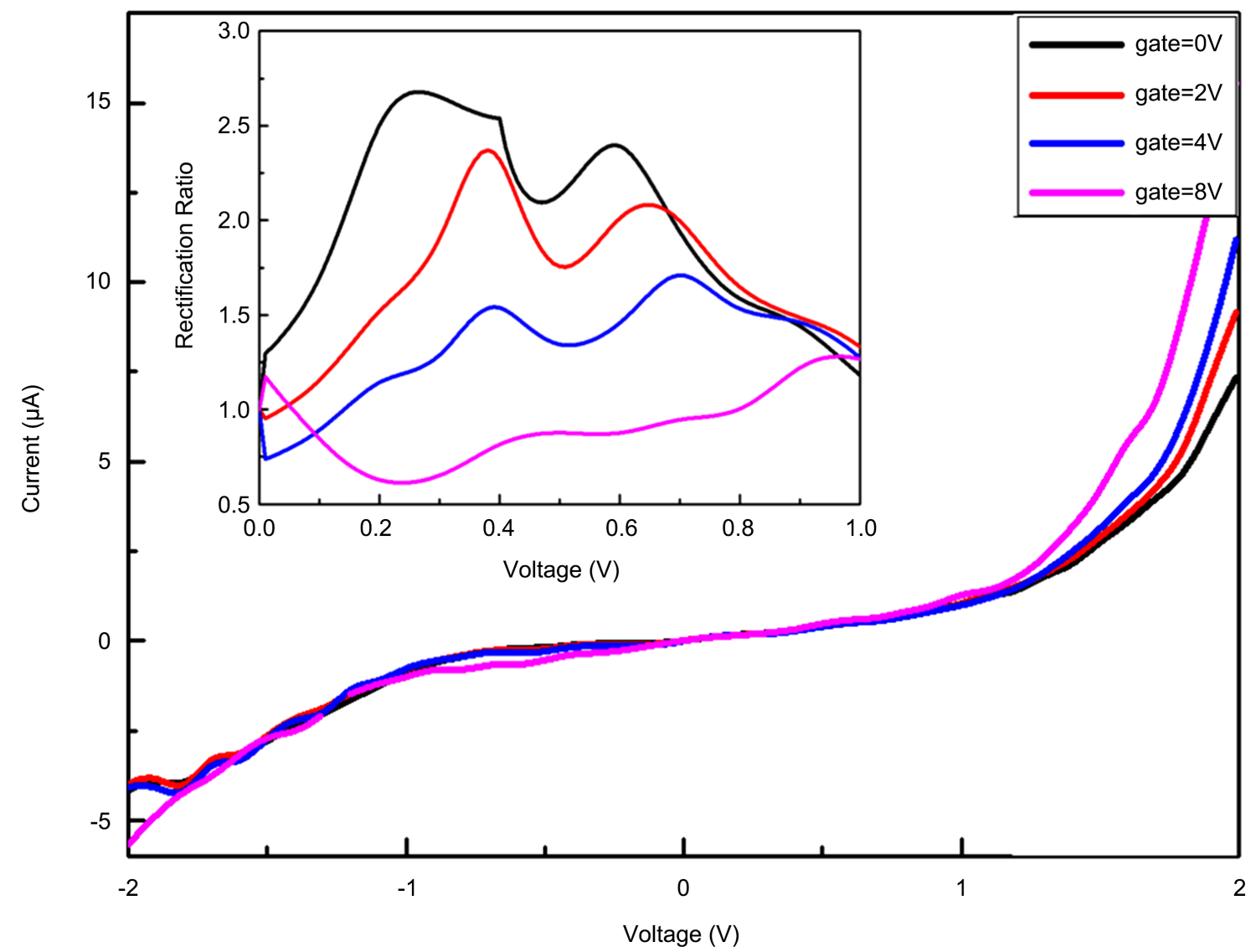

Figure 4. (Color online) Source-Drain currents as a function of $V_{s d}$ for $V_{g}=0.0 \mathrm{~V}, 2.0 \mathrm{~V}, 4.0 \mathrm{~V}$, and $8.0 \mathrm{~V}$, respectively in the M2 device configuration. The inset shows the changes of the corresponding rectification ratio with the applied bias. 
bias due to the currents are almost the same. The inserted figure shows the rectification ratio, a ratio of the currents under positive and negative voltages for the same bias magnitude $(R R=I(V) /|I(-V)|)$. The forward (backward) rectification is defined according to the rectification ratio $R>1(R<1)$. Obviously, while $0.0 \mathrm{~V}<V_{s d}<0.8 \mathrm{~V}$, the rectification ratios are depressed gradually as the gates adding, and the biggest rectification ratio reaches 2.7 at a bias of $0.3 \mathrm{~V}$ as $V_{g}=$ $0.0 \mathrm{~V}$. While $V_{s d}>0.8 \mathrm{~V}$, the rectification ratios are increased rapidly and intersect with the curve of $V_{g}=0.0 \mathrm{~V}$ as the added gates, and the rectification ratio reaches 2.8 at a bias of $2.0 \mathrm{~V}$ with $V_{g}=8.0 \mathrm{~V}$. From this figure we can also see the rectification direction is inversed within the bias region of $[0.06 \mathrm{~V}, 0.8 \mathrm{~V}]$ at the $V_{g}=8.0 \mathrm{~V}$.

To understand the observed $I$ - $V$ curves and the rectification behaviors, we give the transmission spectrum $T(E)$ and molecular projected self-consistent Hamiltonian (MPSH) of four frontier molecular orbitals HOMO-1, HOMO (the highest occupied molecular orbital), LUMO (the lowest unoccupied molecular orbital), and LUMO+1 at $V_{s d}=0.0 \mathrm{~V}$ for different $V_{g}$, as shown in Figure 5. We can see there are broaden transmission peaks around the Femi level invariant with gate voltage. The transmission coefficients at $E_{f}$ are $0.0108,0.0154,0.0206$ and 0.01563 for $V_{g}=0.0 \mathrm{~V}, 2.0 \mathrm{~V}, 4.0 \mathrm{~V}$ and $8.0 \mathrm{~V}$, respectively, so the currents vary approximately linearly in low $V_{s d}$ regions. It is notable that the HOMO resonances are closer to $E_{f}$ therefore, $\mathrm{HOMO}$ is the main transmission channel in this device. Table 1 illustrates the distribution and the eigenvalues of the MPSH of the HOMO-1, HOMO, LUMO and LUMO+1 under zero bias voltage. It can be seen the HOMO state distributes largely in the left region, which means more overlaps of the orbitals between molecule and the left electrode. Following previous investigations, we can conclude that the HOMO orbital moves down at positive bias, instead it will move up at negative bias. Consequently, a slightly smaller negative bias is necessary to drive the HOMO into the bias window. As we can see in this table, though the HOMO resonance is slightly localized, it also verified the small and little-distinction currents in low $V_{s d}$ regions. Although LUMO and LUMO+1 orbitals are always fully delocalized, for they are far away from $E_{f}$ and cannot be excited in low $V_{s d}$ regions to transport electrons.

Figure 6 displays a series of transmission spectra to explore the details of the rectifying performance. As shown in Figure 6(a), there are three transmission peaks always stay in the bias window under the positive bias, but the transmission peaks become significant low when they are under the negative bias. Hence, a forward rectification can be observed in this situation. As shown in Figure 6(b) and Figure 6(c), the conditions are similar to Figure 6(a), there are more and higher transmission peaks in the bias window under the positive bias than the negative bias. With regard to Figure $6(\mathrm{~d})$, at a bias of $\pm 0.4 \mathrm{~V}$ with a $V_{g}=8.0$ $\mathrm{V}$, the case is opposite to the former cases, there are more broaden and higher transmission peaks in the bias window under the negative bias and only two sharp and lower transmission peaks under the positive bias, which means exhibiting a reverse rectification under this bias. 


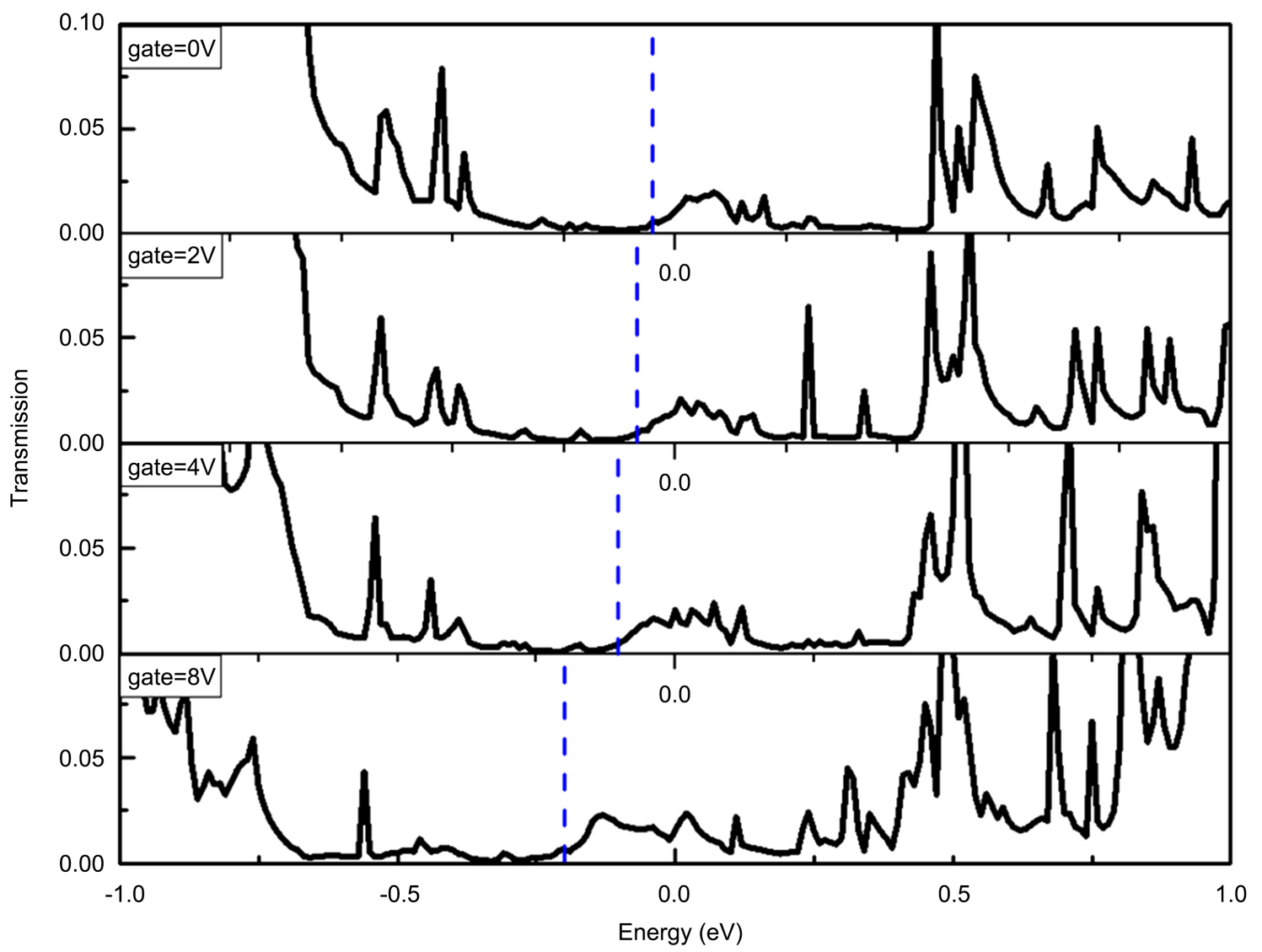

Figure 5. Transmission spectra $\mathrm{T}(\mathrm{E})$ at $V_{s d}=0.0 \mathrm{~V}$ for $V_{g}=0.0 \mathrm{~V}, 2.0 \mathrm{~V}, 4.0 \mathrm{~V}$, and $8.0 \mathrm{~V}$, respectively in the $\mathrm{M} 2$ device configuration.

Table 1. MPSH of four frontier molecular orbitals for $V_{g}=0.0 \mathrm{~V}, 2.0 \mathrm{~V}, 4.0 \mathrm{~V}$, and $8.0 \mathrm{~V}$, respectively, at $V_{s d}=0.0$ $\mathrm{V}$ in the M2 device configuration.

\begin{tabular}{|c|c|c|c|c|}
\hline$V_{g}$ & HOMO-1 & HOMO & LUMO & LUMO+1 \\
\hline $0 \mathrm{~V}$ & $-1.58 \mathrm{eV}$ & $-0.04 \mathrm{eV}$ & $1.58 \mathrm{eV}$ & $2.28 \mathrm{eV}$ \\
\hline $2 \mathrm{~V}$ & $-1.64 \mathrm{eV}$ & $-0.07 \mathrm{eV}$ & $1.52 \mathrm{eV}$ & $2.23 \mathrm{eV}$ \\
\hline $4 \mathrm{~V}$ & $-1.70 \mathrm{eV}$ & $-0.10 \mathrm{eV}$ & $1.46 \mathrm{eV}$ & $2.17 \mathrm{eV}$ \\
\hline $8 \mathrm{~V}$ & $-1.85 \mathrm{eV}$ & $-0.19 \mathrm{eV}$ & $1.29 \mathrm{eV}$ & $2.02 \mathrm{eV}$ \\
\hline
\end{tabular}



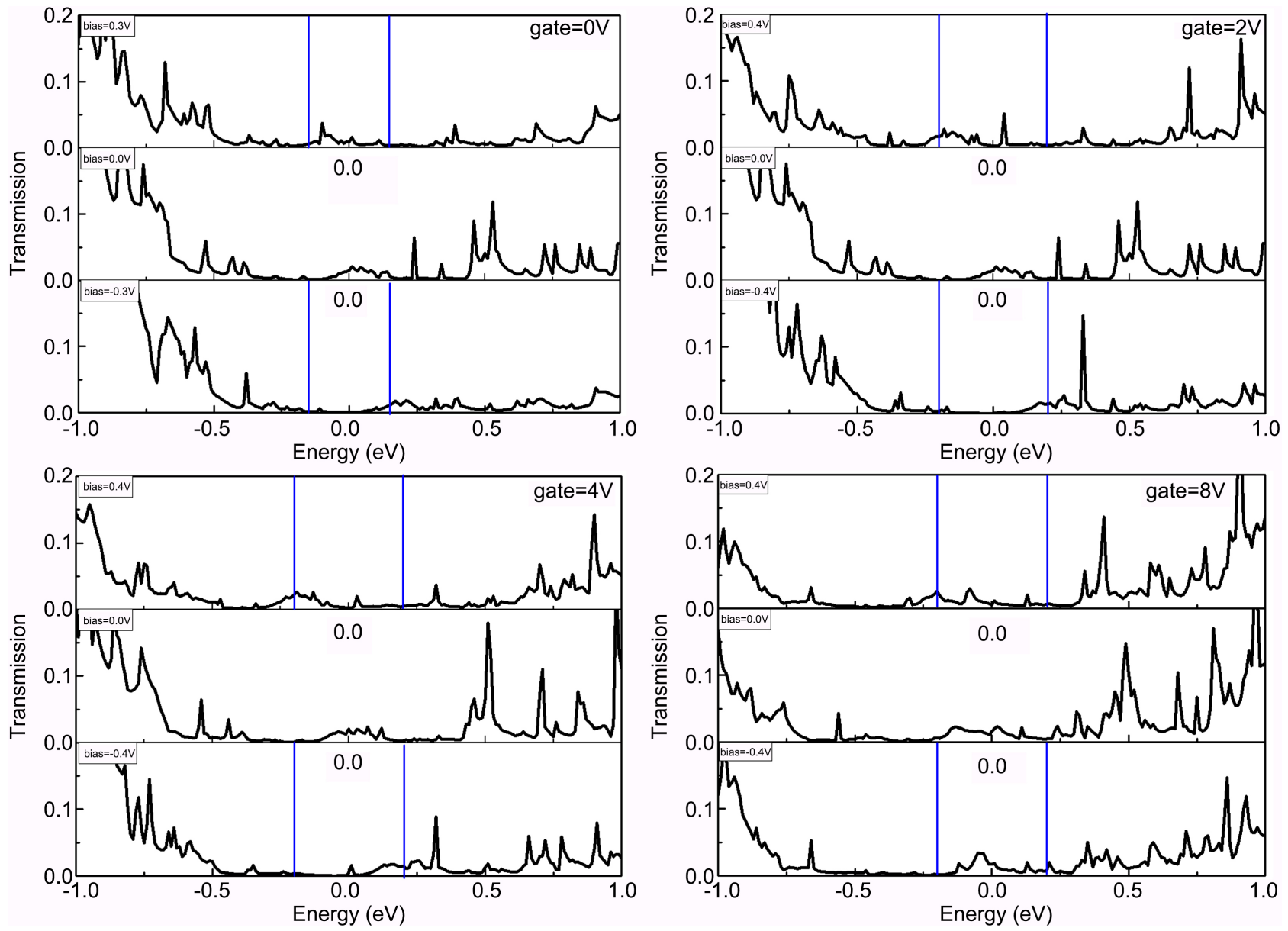

Figure 6. Transmission spectra $\mathrm{T}$ (E) at (a) $V_{g}=0.0 \mathrm{~V}$ for $V_{s d}=0.0 \mathrm{~V}, \pm 0.3 \mathrm{~V}$, and (b) (c) (d) $V_{g}=2.0 \mathrm{~V}, 4.0 \mathrm{~V}, 8.0 \mathrm{~V}$ for $V_{s d}=0.0$ $\mathrm{V}, \pm 0.4 \mathrm{~V}$, respectively.

\section{Summary}

In conclusion, we have investigated the electronic transport properties of phenalenyl molecular with two different contact geometries via different gates. The theoretical results show that the $I$ - $V$ curves are symmetric and the currents are depressed as the applied gates adding with the M1 model. While with the asymmetric M2 model, the currents are asymmetric and the rectification behavior occurs, which means the asymmetric structure will lead to rectification phenomenon, and the rectifying behavior is different as the gates adding, all these findings could be helpful for application of the phenalenyl molecular in the future.

\section{Acknowledgements}

This work was supported by the Natural Science Foundation of Shandong Province (No.ZR2018LA012) and Doctoral Research Start-up Fund (No.2017BSZX03).

\section{Conflicts of Interest}

The authors declare no conflicts of interest regarding the publication of this paper. 


\section{References}

[1] Aviram, A. and Ratner, M.A. (1974) Molecular Rectifiers. Chemical Physics Letters, 29, 277-283. https://doi.org/10.1016/0009-2614(74)85031-1

[2] Lopez-Bezanilla, A., Huang, J., Terrones, H. and Sumpter, B.G. (2011) Boron Nitride Nanoribbons Become Metallic. Nano Letters, 11, 3267-3273.

https://doi.org/10.1021/nl201616h

[3] Liu, N., Gao, G.Y., Zhu, S.C., Ni, Y., Wang, S.L., Liu, J.B. and Yao, K.L. (2014) Carbon Doping Induced Peculiar Transport Properties of Boron Nitride Nanoribbons $p-n$ Junctions. Journal of Applied Physics, 116, Article ID: 023708. https://doi.org/10.1063/1.4890225

[4] Jrvinen, P., Hmlinen, S.K., Banerjee, K., Häkkinen, P., Ijäs, M., Harju, A., et al. (2013) Molecular Self-Assembly on Graphene on $\mathrm{SiO}_{2}$ and h-BN Substrates. Nano Letters, 13, 3199-3204. https://doi.org/10.1021/nl401265f

[5] Kharche, N. and Nayak, S.K. (2011) Quasiparticle Band Gap Engineering of Graphene and Graphone on Hexagonal Boron Nitride Substrate. Nano Lett, 11, 5274-5278. https://doi.org/10.1021/nl202725w

[6] Liang, Y., Xin, W., Juan, W., Cui, G. and Liu, D. (2018) Graphite Carbon Nitride/Boron Doped Graphene Hybrid for Efficient Hydrogen Generation Reaction. Nanotechnology, 29, Article ID: 345705. https://doi.org/10.1088/1361-6528/aac9ae

[7] Zhao, J., Zhao, W., Cui, B., Fang, C., Xu, Y., Kong, X., et al. (2014) Electronic Transport Properties of a Dithienylethene-Based Polymer with Different Metallic Contacts. RSC Advances, 4, 40941-40950. https://doi.org/10.1039/C4RA06904H

[8] Zhao, J., Fang, C., Cui, B., Zou, D., Zhao, W., Li, X., et al. (2016) Spin Transport Properties in Silicene-Based Heterojunctions with Different Edge Hydrogenation. Organic Electronics, 41, 333-339. https://doi.org/10.1016/j.orgel.2016.11.025

[9] Fan, Z.Q. and Chen, K.Q. (2010) Negative Differential Resistance and Rectifying Behaviors in Phenalenyl Molecular Device with Different Contact Geometries. Applied Physics Letters, 96, Article ID: 053509. https://doi.org/10.1063/1.3309708

[10] Li, H., Wang, L., Liu, Q., Zheng, J., Mei, W.-N., Gao, Z., et al. (2012) High Performance Silicone Nanoribbon Field Effect Transistors with Current Saturation. European Physical Journal B, 85, Article No. 274. https://doi.org/10.1140/epjb/e2012-30220-2

[11] Tombros, N., Jozsa, C., Popinciuc, M., Jonkman, H.T. and van Wees, B.J. (2007) Electronic Spin Transport and Spin Precession in Single Grapheme Layers at Room Temperature. Nature, 448, 571-574. https://doi.org/10.1038/nature06037

[12] Van-Truong, T., Jérôme, S.-M. and Philippe, D. (2014) Large on/off Current Ratio in Hybrid Graphene/BN Nanoribbons by Transverse Electric Field-Induced Control of Bandgap. Applied Physics Letters, 105, Article ID: 073114. https://doi.org/10.1063/1.4893697

[13] Kim, J., Lee, W., Choi, S., Kim, K.-T. and Kim, Y.-H. (2020) Dual-Gate Crystalline Oxide-Nanowire Field-Effect Transistors Utilizing Ion-Gel Gate Dielectric. Applied Surface Science, 515, Article ID: 145988. https://doi.org/10.1016/j.apsusc.2020.145988

[14] Jian, p. and Wang, Q (2020) Investigating Electrical Properties of Controllable Graphene Nanoribbon Field Effect Transistors. Physica B: Condensed Matter, 583, Article ID: 412022. https://doi.org/10.1016/j.physb.2020.412022

[15] Keren, K., Berman, R.S., Buchstab, E., Sivan, U. and Braun, E. (2003) DNA-Templated Carbon Nanotube Field-Effect Transistor. Science, 302, 1381-1382. 
https://doi.org/10.1126/science.1091022

[16] Song, H., Kim, Y., Jang, Y.H., Jeong, H.J., Reed, M.A. and Lee, T.H. (2009) Observation of Molecular Orbital Gating. Nature, 462, 1039-1043.

https://doi.org/10.1038/nature08639

[17] Li, L., Yu, Y., Ye, G.J., Ge, Q., Ou, X., Wu, H., et al. (2014) Black Phosphorus Field-Effect Transistors. Nature Nanotechnology, 9, 372-377. https://doi.org/10.1038/nnano.2014.35

[18] Xu, B., Xiao, X., Yang, X. and Tao (2005) Large Gate Modulation in the Current of a Room Temperature Single Molecule Transistor. Journal of the American Chemical Society, 127, 2386-2387. https://doi.org/10.1021/ja042385h

[19] Singh, A., Khosla, M. and Raj, B. (2019) Design and Analysis of Dynamically Configurable Electrostatic Doped Carbon Nanotube Tunnel FET. Microelectronics Journal, 85, 17-24. https://doi.org/10.1016/j.mejo.2019.02.001

[20] Priscilla Scarlet, S., Ambika, R. and Srinivasan, R. (2017) Effect of Eccentricity on Junction and Junctionless Based Silicon Nanowire and Silicon Nanotube FETs. Superlattices and Microstructures, 107, 178-188. https://doi.org/10.1016/j.spmi.2017.04.015

[21] Zheng, J., Wang, L., Ruge, Q., Liu, Q., Li, H., Yu, D., et al. (2013) Sub-10nm Gate Length Graphene Transistors: Operating at Terahertz Frequencies with Current Saturation. Scientific Reports, 3, Article No. 1314. https://doi.org/10.1038/srep01314

[22] Eldon, E. and George, K. (2000) Principles for the Design and Operation of a Molecular Wire Transistor. Journal of Applied Physics, 88, Article No. 5280. https://doi.org/10.1063/1.1315627

[23] Di Ventra, M. and Pantelides, S.T. (2000) The Benzene Molecule as a Molecular Resonant-Tunneling Transistor. Applied Physics Letters, 76, Article No. 3448. https://doi.org/10.1063/1.126673

[24] Yang, Z. and Lang, N.D. (2003) Effects of Geometry and Doping on the Operation of Molecular Transistors. Applied Physics Letters, 82, 1938-1940. https://doi.org/10.1063/1.1563737

[25] Ghosh, A.W., Rakshit, T. and Datta, S. (2004) Gating of a Molecular Transistor: Electrostatic and Conformational. Nano Letters, 4, 565-568. https://doi.org/10.1021/nl035109u

[26] Britnell, L., Gorbachev, R.V., Jalil, R. and Belle, B.D. (2012) Field-Effect Tunneling Transistor Based on Vertical Graphene Heterostructures. Science, 335, 947-950.

[27] Takano, Y., Taniguchi, T., Isobe, H., Kubo, T., Morita, Y., Yamamoto, K., et al. (2002) Hybrid Density Functional Theory Studies on the Magnetic Interactions and the Weak Covalent Bonding for the Phenalenyl Radical Dimeric Pair. Journal of the American Chemical Society, 124, 11122-11130. https://doi.org/10.1021/ja0177197

[28] Huang, J. and Kertesz, M. (2007) Intermolecular Covalent $\pi-\pi$ Bonding Interaction Indicated by Bond Distances, Energy Bands, and Magnetism in Biphenalenyl Biradicaloid Molecular Crystal. Journal of the American Chemical Society, 129, 16341643. https://doi.org/10.1021/ja066426g

[29] Pal, S.K., Itkis, M.E., Tham, F.S., Reed, R.W., Oakley, R.T. and Haddon, R.C. (2008) Trisphenalenyl-Based Neutral Radical Molecular Conductor. Journal of the American Chemical Society, 130, 3942-3951. https://doi.org/10.1021/ja0777710

[30] Fan, Z.Q., Sun, W.Y., Zhang, Z.H., Deng, X.Q., Tang, G.P. and Xie, H.Q. (2017) Symmetry-Dependent Spin Transport Properties of a Single Phenalenyl or Pyrene Molecular Device. Carbon, 122, 687-693. 
https://doi.org/10.1016/j.carbon.2017.07.019

[31] Wang, L., Tagami, K. and Tsukada, M. (2004) Quantum Transport through Multiterminal Phenalenyl Molecular Bridges. Japanese Journal of Applied Physics, 43, 2779. 